\title{
EXTERNAL EVIDENCE IN THE VALIDATION OF MENTALISTIC THEORIES : A CHOMSKYAN PARADOX*
}

RUDOLF P. BOTHA

\section{Introduction}

Is it "legitimate" and "proper" to "impute existence to" or "attribute psychological reality to" the theoretical constructs of linguistic theories? This is one of the main questions considered by Professor Chomsky in his article "On the biological basis of language capacities" (1976). Chomsky's discussion of this question may be seen as an attempt to clarify and justify the methodological bases of mentalistic linguistics. That these methodological bases are in need of clarification and justification has been pointed out over the years by various scholars. (I) Moreover, Katz (1977:564) has recently admitted that he does not fully understand Chomsky's position on the psychological reality of grammars. This admission by Katz is particularly significant. (2) For, recall that he has co-authored with Chomsky a paper in which they attempt, among other things, to explicate the sense in which grammars may be claimed to be psychologically real. (3)

The present paper critically analyzes one aspect of the above-mentioned attempt by Chomsky at clarifying and justifying the methodological bases of mentalistic linguistics. To be more specific: the object under critical scrutiny is Chomsky's position on the nature of the evidence pertinent to the validation of mentalistic (linguistic) theories. This position will be reconstructed in terms of a number of evidential theses at the basis of which lies Chomsky's view that external (linguistic) evidence can play only a limited role in this validation. It is argued that this view of Chomsky's gives rise to serious doubts about the empirical nature of his mentalistic theories, grammatical as well as general linguistic. The obvious way of removing these doubts, it is then argued, leads to the identification of a paradox in Chomsky's mentalistic-rationalistic approach to the study of language. The final conclusion of the paper is that this paradox stands in the way of agreeing that it is "legi- 
timate" and "proper" for Chomsky to "impute existence to" theoretical linguistic constructs. The argument outlined above is developed in 8\$6-9 below against the background sketched in $\$ \S_{2}-5$.

\section{Chomskyan mentalism}

To see what it is all about, consider the sentence (I) and the questions (2) and (3).

(1) Violins are easy to play sonatas on,

(2) What violins are easy to play sonatas on?

(3) What sonatas are violins easy to play on?

To illustrate the method of mentalistic linguistics, Chomsky (1976:7) considers the following problem in connection with these expressions: Why is it that (3), unlike (2), is not well-formed as a question corresponding to (1)? Chomsky's (1976:7-9) tentative solution to this problem boils down to the following: wh-clauses are "islands" in the sense that a rule such as wh-movement --- which forms questions and relatives by moving such expressions as who, what, what sonatas, etc., to the left of a clause --cannot be applied in general to a (second) wh-expression within a wh-clause. At the stage where wh-movement applies in the derivation of the question (3), sonatas is a constituent of a wh-clause which may be represented as follows:

(4) $[S \text { which for PRO to play sonatas on } t]^{(4)}$

Thus, in the derivation of ( 3 ), wh-movement moves a "questioned" constituent, sonatas, out of a wh-clause, viz. an infinitival relative; and by so doing it violates the wh-island constraint. Consequently, the resulting question has to be ungrammatical. (5) Let us call this explanation for the sake of later reference "the/Chomsky's wh-explanation".

At the surface, this explanation of the ungrammaticalness of (3) appears to be a fragment of a straightforwardly nonmentalistic and formal grammar. A lawlike linguistic generalization, together with a number of specific fact-asserting statements, functions as the explanans. From these a 
statement describing a problematic phenomenon -- the ungrammaticalness of (3) -- is deduced as the explanandum. Chomsky, however, takes a further step: he "imputes existence to" the theoretical constructs involved in the explanans. Thus he (1976:9) states that: "Tentatively accepting this explanation, we impute existence to certain mental representations and to the mental computations that apply in a specific way to these mental representations. In particular, we impute existence to a representation in which (12) $[=$ our (4) above --- R.P.B. $]$ appears as part of the structure underlying (5). $[=$ our (3) above --- R.P.B. $]$ at a particular stage of derivation, and to the mental computation that produces this derivation, and ultimately produces (5), identified now as ungrammatical because the computation violates the wh-island constraint when the rule of wh-movement applies to sonatas in (12). We attribute 'psychological reality' to the postulated representations and mental computations. In short, we propose (tentatively, hesitantly, etc.) that our theory is true. Have we gone beyond the bounds of what is legitimate and proper, in so doing?" By imputing existence to its theoretical constructs, Chomsky transforms a fragment of nonmentalistic, formal grammar into a fragment of mentalistic grammar. (6) And the crucial question, raised by Chomsky himself, is whether this is or is not objectionable.

Chomsky's reply to this question is in the negative. A proper answer can only, however, be given against the background of a clear and principled account of the methodological bases of mentalistic linguistics. Such an account will provide proper answers to questions like the following:

(5) (a) What are the objects in the real world which mentalistic (linguistic) theories -- grammars as well as general theories -- are theories about?

(b) What are the aims which these theories pursue in regard to the objects in question?

(c) What is the epistemological status --- empirical or nonempirical -- which the claims expressed by mentalistic theories are supposed to have?

(d) What is the logic which is required for the validation -- i.e., confirmation and refutation --- of these mentalistic claims? 
Collectively, Chomsky's answers to these and related questions constitute what this paper calls "Chomskyan mentalism". (7) Let us consider these answers as they are given, implicitly or explicitly, in Chomsky's abovementioned article, and then return to the question of whether or not his imputing existence to the theoretical constructs in question is in fact "legitimate and proper".

\section{Objects and aims of mentalistic theories}

Chomsky deals in a quite direct manner with questions (5)(a) and (b), thereby identifying the objects and aims of mentalistic theories.

As regards the general theory, or universal gramar, Chomsky follows Lenneberg in characterizing its object of study as "innate mechanisms, an underlying biological matrix that provides a framework within which the growth of language proceeds" (1976:2); as "the genetic program that enables the child to interpret certain events as linguistic experience and to construct a system of rules and principles on the basis of this experience" (1976:2-3); as "the genetically determined program that specifies the range of possible grammars for human languages" (1976:13). The aim selected by Chomsky (1976:2) for the general theory is to give "an abstract partial specification" of the object specified above.

As regards (particular) grammars, Chomsky once again follows Lenneberg in characterizing their object of study as "a component in the system of cognitive structures" (1976:2); as "a.steady state of mind" (1976:3); as "a mental organ" (1976:3); as "the particular realizations of this schematism [i.e., the genetic program which makes language growth possible --- R.P.B.] that arise under given conditions" (1976:13). The aim of (particular) grammars is described by Chomsky (1976:3) as that of giving "a partial characterization" of the object identified above.

Consider, in summary, the following integrated account provided by Chomsky (1976:3) of the objects and aims of mentalistic theories: "To put the matter in somewhat different but essentially equivalent terms, we may suppose that there is a fixed, genetically determined initial state of mind, common to the species with at most minor variation apart from pathology. The mind passes through a sequence of states under the boundary conditions 
set by experience, achieving finally a 'steady state' at a relatively fixed age, a state which then changes only in marginal ways. The initial state of mind might be regarded as a function, characteristic of the species, which maps experience into the steady state. Universal grammar is a partial characterization of this function, thus a partial characterization of the initial state. The grammar of a language that has grown in the mind is a partial characterization of the steady state attained. So viewed, linguistics is the abstract study of certain mechanisms, their growth and maturation."

In regard to this account of the objects and aims of mentalistic theories, a further question should be considered here: What exactly are the ways in which the characterizations offered by mentalistic theories of their objects are "abstract" and "partial"?

First, as pointed out by Chomsky (1976:3-4), these characterizations are "abstract" in the sense that they idealize their objects. In the actual process of language acquisition or growth the cognitive system characterized by the general theory interacts with other cognitive systems. Similarly, in actual linguistic performance the cognitive system characterized by a particular grammar also interacts with other cognitive systems. Neither the general theory nor a particular grammar, however, aims to give an account of this interaction. By so doing these mentalistic theories abstract away from the contribution of the other cognitive systems which interact with their respective objects, viz. the child's language acquisition faculty and the speaker's linguistic competence. In a nonabstract characterization of this faculty and this competence, it will also be specified how these two cognitive systems interact with other cognitive systems. (8)

Second, according to Chomsky (1976:9) the characterizations offered by the general theory and particular grammars of their respective objects consist of "abstract conditions that unknown mechanisms must meet". That is, it is not the case that these characterizations describe "actual mechanisms" "functioning in the brain", to use Chomsky's terminology. By implication, a nonabstract characterization of the cognitive systems in question will, somehow, specify "actual mechanisms". 
The characterizations which mentalistic theories offer of their objects are, then, abstract in a dual sense: in the sense of "abstracting away from the contribution of other cognitive systems", and in the sense of "being not descriptive of actual mechanisms". To the extent that these characterizations are "abstract", they are "partial" as well. (9)

\section{Epistemological status and logic of validation of mentalistic theories}

This brings us to the epistemological status and the logic of validation of mentalistic linguistic theories, i.e. to questions (5)(c) and (d), respectively. As regards the question of epistemological status, Chomsky (1976: $3,10,20)$, once again following Lenneberg, repeatedly stresses the point that the claims made by mentalistic theories are "empirical". Thus, Chomsky (1976:20) states with regard to the general theory that "... Lenneberg was quite right to take the trouble to emphasize that 'the discovery and description of innate mechanisms is a thoroughly empirical procedure and is an integral part of modern scientific inquiry', and to insist that there is no room here for dogmatism or a priori doctrine". From these and other, similar, remarks by Chomsky on the epistemological status of mentalistic theories, we may draw the following conclusion: If the imputation of existence to theoretical linguistic constructs were to yield nonempirical mentalistic claims, then this imputation of existence would have to be "illegitimate" and "improper" for Chomsky.

Several important questions arise at this stage: What is the content of Chomsky's notion "empirical"? When analyzed within a principled philosophical framework, is this content free of objectionable aspects? A direct approach to these and related questions would, to begin with, entail carrying out three sorts of steps. The first of these would be to specify explicitly the conditions which scientific statements in general have to meet in order to qualify as "empirical". The second would be to give a justification for a particular choice of conditions on empiricalness from among the alternatives discussed in the literature. (10) The third would be to show that the mentalistic claims made by Chomsky do in fact meet the conditions chosen.

In the article under consideration, unfortunately, Chomsky has not adopted 
such a direct approach to providing a clarification of and justification for the content of his notion "empirical". I know of no principled philosophical context within which it is other than wholly uninformative to say that "empirical" means "nondogmatic" and "non a priori". Thus, Chomsky fails to deal explicitly and directly with the question (5)(c) of the epistemological status of mentalistic theories. (1I) And this is the case with question (5)(d) as well: Chomsky does not, within some principled metascientific framework, deal explicitly and directly with the conditions that have to be met by the logic of validation of mentalistic theories.

Rather Chomsky adopts an indirect approach to questions (5)(c) and (d). Specifically, he takes a number of indirect steps to clarify and justify the empirical status and logic of validation of mentalistic theories. Firstly, he constructs an analogy between linguistic inquiry and a particular form of physical inquiry, viz. astrophysical inquiry. Second, and once again with the aim of clarifying and justifying the logic of validation of his version of mentalism, Chomsky constructs an analogy between what mentalist linguists and psychologists do and what certain biologists and neurophysiologists are claimed to do. Thirdly, Chomsky presents a case against the position of those scholars who have criticized his mentalistic approach for having an evidential basis which is insufficiently wide. I have argued elsewhere that both of the former analogies have such defects that, in stead of contributing to Chomsky's attempt to justify his version of mentalism, they draw the attention to a number of methodological defects of this approach. (12) So let us take a closer look at Chomsky's position on the role of external evidence in the validation of mentalistic theories.

\section{Two fundamental distinctions}

To start with, we consider two fundamental distinctions with reference to which this position has to be analyzed. The first distinction pertinent to an analysis of Chomsky's position on the status of external evidence is that between intuitive and nonintuitive (linguistic) evidence. Chomskyans assign linguistic intuitions or so-called informant judgments the status of primary linguistic data. (13) These intuitions play a dual methodological 
role in linguistic inquiry. On the one hand, they constitute problematic data to be explained by particular grammars. On the other hand, they constitute the basic source of evidence for validating --- that is, justifying and refuting --- such grammars. It has become conventional to denote the evidence yielded by linguistic intuitions -- or, in Chomsky's (1976:12) terms, the "evidence derived from informant judgment" -- by means of the expression "internal (linguistic) evidence". By contrast, nonintuitive linguistic evidence, of all kinds, is referred to by means of the generic term "external evidence". Internal evidence consists in data about the objects internal to the generative grammarian's linguistic reality as this reality is delineated by means of the abstractions and idealizations employed by him. (14) External evidence, by contrast, consists in data about phenomena, objects or processes which, in terms of these same abstractions and idealizations, are external to this linguistic reality. External evidence comprises, for instance, data about the physical basis of the language capacity, data about the actual use of linguistic competence in performance, data about the genetic basis of the language capacity, data about linguistic change, data about speech pathology, etc.

The second distinction is the one between a mentalistic and a nonmentalistic (fragment of a) linguistic theory such as a grammar. This distinction may be elucidated with reference to Chomsky's wh-explanation outlined in $\mathrm{S}_{2}$ above. Observe that this wh-explanation is not inherently mentalistic: it is a straightforward fragment of formal, nuts-and-bolts grammar. Specifically, it makes no ontological claims about any underlying reality, whether mental or other. As presented above, the wh-explanation thus incorporates no element in virtue of which a nonmentalist linguist would be unable to present it as a potential solution to the problem of the ungrammaticalness of (3). This wh-explanation is simply an ordered set of statements: some of these collectively constitute the explanans from which a statement describing the problematic ungrammaticalness of (3) can be derived as the explanandum. The view of linguistic theories called by Katz (1977) "Platonism" or the "Platonist Position" may be taken to represent one form of nonmentalism. According to Platonism, "grammar is an abstract science like arithmetic"(p.562). A Platonist grammar does not characterize real 
entities such as idealized mental objects or processing systems (pp.565-6). It rather depicts "the structure of abstract entities" (p.566).

In order to turn the inherently nonmentalistic wh-explanation into a fragment of mentalistic grammar, Chomsky has to add a number of claims to those already incorporated in this explanation. The claims which Chomsky adds to the wh-explanation are those by means of which he (1976:9) "imputes existence to" or "attributes psychological reality to" the "mental representation" (4) and the "mental computations" involved in the derivation of the question (3). These ontological claims made by Chomsky may, for short, be called "mentalistic claims/hypotheses". In sum: a nonmentalistic linguistic theory does not aim at describing a real object, mental or other; a mentalistic linguistic theory, by contrast, has the aim of describing the structure of a mental object or entity.

Against this background, it is now possible to examine Chomsky's position on the role of external evidence in the validation of mentalistic linguistic theories. I will attempt to explicate this position of Chomsky's in terms of four evidential theses. These represent my reconstruction of Chomsky's position --- he makes no attempt at giving an explicit account of his position.

\section{The sources of evidence}

A first aspect of Chomsky's position on the evidence pertinent to the validation of mentalistic theories, and in particular grammars, may be reconstructed as follows.

(6) The Varied Sources Thesis: Evidence bearing on mentalistic claims may be derived from many and varied sources.

The Varied Sources Thesis represents the core of the following remarks by Chomsky (1976:3): "We may impute existence to the postulated structures at the initial, intermediate, and steady states in just the same sense as we impute existence to a program that we believe to be somehow represented in a computer or that we postulate to account for the mental representation of a three-dimensional object in the visual field. Evidence bearing 
on empirical hypotheses such as these might derive from many and varied sources. Ultimately, we hope to find evidence concerning the physical mechanisms that realize the program, and it is reasonable to expect that results obtained in the abstract study of the system and its operation should contribute significantly to this end (and in principle, conversely)". The "initial state" mentioned in this quote represents "a fixed, genetically determined initial state of mind common to the species" that makes language acquisition or "growth" possible, that is the so-called faculté de langage or language acquisition device. The "steady state" represents "the grammar of a language that has grown in the mind", i.e. the idealized linguistic competence of the adult speaker.

The Varied Sources Thesis -- implicitly adopted by Chomsky in earlier work as well (15) -- appears at the surface to be nonobjectionable. Clearly, the more numerous and the more varied the sources of evidence for mentalistic claims were, the more thorough would be the validation of these hypotheses. Moreover, what point could there be to restricting the evidence for mentalistic hypotheses to a single source, viz. native speaker intuitions? In spite of these obviously attractive aspects of The Varied Sources Thesis, it is problematic within the wider context of Chomskyan generative grammar. The problems spring from the abstractions and corresponding idealizations employed by Chomsky. Let us consider two of these idealizations as they bear on the study of the cognitive system known as "Iinguistic competence".

The first abstraction and corresponding idealization concern the manner in which other cognitive systems interact with linguistic competence in the actual use of language. Thus, Chomsky (1976:3) points out that "When we speak or interpret what we hear, we bring to bear a vast set of background assumptions about the participants in the discourse, the subject matter under discussion, laws of nature, human institutions, and the like". He proceeds (1976:3-4) to point out that "In an effort to determine the nature of one of these interacting cognitive system [i.e., linguistic competence --- R.P.B.], we must abstract away from the contribution of others [such as the cognitive system of background assumptions --- R.P.B.] to the actual performance that can be observed".

This abstraction, and the resulting idealization, have two complementary 
consequences. On the one hand, because of this abstraction problematic data about the contribution which the above-mentioned "other" cognitive systems make towards actual performance are excluded from the domain of problematic data to be accounted for by the mentalist grammarian. On the other hand, this abstraction stipulates that such data are irrelevant in principle to the validation of' mentalistic hypotheses about an idealized competence. For example, by abstracting away from the manner in which a speaker's background knowledge interacts with his competence in actual performance, a mentalist linguist, as a matter of principle, stipulates that data about this knowledge and about its interaction with competence are irrelevant to the validation of mentalistic hypotheses about the idealized competence.

The second abstraction yields an idealization known as "the ideal speakerlistener". Chomsky (1965:3) states that "Linguistic theory is concerned primarily with an ideal speaker-listener in a completely homogeneous speech-community, who knows its language perfectly and is unaffected by such grammatically irrelevant conditions as memory limitations, distractions, shifts of attention and interest, and errors (random or characteristic) in applying his knowledge of the language in actual performance". In terms of this idealization, the linguistic reality studied by the mentalist includes pure, idealized competence alone, excluding such phenomena, objects or processes as those involved in idiolectal, dialectal and sociolinguistic variation, in the production and perception of utterances, in linguistic change, in speech pathology and errors, in pidginization and creolization, etc. Here, too, abstracting away from these phenomena, objects or processes has two complementary consequences. On the one hand, data about these phenomena, objects or processes fall outside the domain of problematic data to be accounted for by menatlistic theories. On the other hand, it is implied that these data are irrelevant in principle to the validation of mentalistic claims about an idealized competence.

Thus, Chomskyan idealizations such as the two mentioned above do not only restrict the domain of problematic data of mentalistic theories. These idealizations restrict in principle the sources of evidence for the validation of mentalistic claims as well. In fact, the sources of potential evidence for mentalistic claims are restricted, as a result of these idealizations, to one only: data about pure linguistic competence, i.e. Iin- 
guistic intuitions of native speakers. Notice, moreover, that not even all intuitive informant judgments about properties of linguistic units qualify as potential evidence for validating mentalistic claims. Only those intuitive judgments which are causal effects of linguistic competence itself are relevant to the validation of mentalistic claims. Scholars such as Bever and Katz have shown that certain intuitive judgments are causal effects not of iinguistic competence, but of such psychological mechanisms as perceptual strategies, etc. (16) In terms of Chomsky's idealizations these intuitive judgments are irrelevant to the validation of mentalistic claims. That Chomsky accepts this consequence is clear from the following remarks of his (1978b:10): "... we often do not know what is the right kind of evidence. When we elicit judgments from informants, or conduct psycholinguistic experiments, we do not know a priori what we should attribute to grammatical competence as distinct from innumerable other factors".

Viewed against the background of the abstractions and idealizations employed by Chomsky, his Varied Sources Thesis is thus all but nonproblematic. The latter thesis allows for "many and varied sources" from which evidence may be derived for the validation of mentalistic claims. The former idealizations, however, restrict these sources to one: genuine linguistic intuitions. It is therefore not strange that Jerrold Katz -- one of the few generative grammarians who has given serious thought to the methodological bases of mentalistic linguistics -- is willing to accept evidence from this one source alone. The core of his (1977:563) position is that "Competencism claims that idealizations in grammar proceed only from intuitions of grammatical properties and relations. Data pertaining to the nature of events in tasks involving high speed operations, such as errors and reaction times, do not enter into the evidential constraints in grammar construction. Such events are different in kind from mental acts of inner apprehension [i.e. linguistic intuitions --R.P.B.] - They reflect aspects of the way speakers exercise their knowledge rather than features of the knowledge itself. Accordingly, the competencist can give a priori grounds for considering the sort of data that FFG [i.e., Fodor, Fodor and Garrett (1975) -- R.P.B.] use to argue their case against semantic representation to be just the sorts of data that a linguist should iEnore in grammar construction ..." (17) The data used by FFG and judged irrelevant by Katz comprise data about the use of semantic representations in performance tasks. From the quote given 
above, it is clear that Katz cannot accept Chomsky's Varied Sources Thesis. The crucial question, then, is how it is possible for Chomsky simultaneousIy to employ the idealizations discussed above and to accept The Varied Sources Thesis. That is: How, within the Chomskyan approach, is it possible for data about phenomena, objects and processes from which these idealizations abstract away to be used for validating mentalistic claims about an idealized linguistic competence? For ease of reference, this problem may be denoted by means of the expression "The Mentalist-Rationalist Paradox". The origin of the term "Mentalist" within this compound expression is obvious. The term "Rationalist" derives from the philosophy of science which underlies the use of the idealizations in question.

\section{The nonnecessity of extemal evidence}

This brings us to Chomsky's second evidential thesis.

(7) The Nonnecessity Thesis: It is not necessary, for the validation of mentalistic claims, to use, in addition to intuitive (= internal) evidence, other, nonintuitive (= external), evidence.

Expressed in The Nonnecessity Thesis is the essence of the following remarks by Chomsky (1976:5-6): "Challenged to show that the constructions postulated in that theory [i.e., a theory about the initial/final state of the language faculty --- R.P.B.] have 'psychological reality', we can do no more than repeat the evidence and the proposed explanations that involve these constructions. Or, like the astronomer dissatisfied with study of light emissions from the periphery of the sun, we can search for more conclusive evidence, always aware that in empirical inquiry we can best support a theory against substantive alternatives and empirical challenge, not prove it to be true". Within this context, the evidence which it is permissible to repeat, according to Chomsky, is intuitive evidence. The "more conclusive evidence" mentioned in the quote has to be nonintuitive, external, evidence. (19) The crucial part of the quote, of course, is Chomsky's use of $\underline{\text { Or }}$ in the statement "Or, like the astronomer dissatisfied with ...". This use of Or clearly indicates that Chomsky does not consider the use of external evidence a necessity in the validation of men- 
talistic claims. (20) Let us now consider a fundamental problem with Chomsky's Nonnecessity Thesis. (2I)

This problem concerns the empirical nature of the mentalistic claims expressed in Chomskyan linguistic theories. It has been shown above that a fragment of grammar such as Chomsky's wh-explanation is not inherently mentalistic. To turn it into a fragment. of mentalistic grammar, a mentalist has to add a number of claims to those already incorporated in this explanation. The claims added to those already incorporated in the wh-explanation have to meet a specific condition: they must be empirical. Otherwise there would be no difference in refutable content between a mentalistic interpretation of this explanation and a nonmentalistic one. In the absence of such a difference in refutable content, the difference between a fragment of mentalistic grammar and a fragment of nonmentalistic grammar would be either terminological or metaphysical.

We have seen in 82 that Chomsky appears to be aware of this difficulty. Though he fails to discuss this problem explicitly, he does make an attempt to add something to the content of the claims incorporated in the wh-explanation. Specifically, what Chomsky (1976:9) does is "to impute existence" to the theoretical constructs in terms of which these hypotheses are formulated. From the quote presented in $\$ 2$, it is clear that Chomsky does this by adding to the claims incorporated in his wh-explanation ontological mentalistic claims such as the following.

(8) (a) $[s$ which for PRO to play sonatas on $t]$ exists as a component part of a mental representation underlying the question What sonatas are violins easy to play on?

(b) wh-movement exists as a component part of the mental computations by means of which the question What sonatas are violins easy to olay on? is derived.

The question, now, is whether or not the ontological claims (8)(a) and (b) add refutable elements of content to the wh-explanation. That is, are the claims (8)(a) and (b), - - and other ontological claims of the same kind -- more than mere verbalisms or metaphysical statements? In other words, do the claims (8)(a) and (b) make a substantive difference in content between Chomsky's mentalistic interpretation of the wh-explanation and a nonmentalistic interpretation of it? 
Mentalistic claims such as (8)(a) and (b) exhibit two problematic properties which indicate that they are nonempirical in the conventional sense. The first property is that of ontological indeterminacy and is dealt with in 87.1 below. The second property, that of evidential indeterminacy, is directly related to the adoption of The Nonnecessity Thesis. Let us, to begin with, consider this property in abstract terms.

Suppose that there were two sets of claims A and B such that B incorporated all the claims of A plus a number of additional claims. If these aditional claims were to be empirical, then there would have to be a difference in the evidence that bore on $A$ and $B$. Thus all the evidence relevant to the validation of $A$ would alșo be relevant to the validation of $B$. But there would have to be additional evidence that bore on $B$ but not on $A$. Specifically, this additional evidence would have to bear on the additional claims of B. Since these claims were not incorporated in A, this additional evidence would simply be irrelevant to the validation of $A$. If it were impossible in principle to bring additional evidence of the appropriate kind to bear on the additional claims of $B$, then these claims would be evidentially indeterminate. This property would render the additional claims nonempirical. This is to say that there is no real empirical difference in the content of the sets of claims A and B despite B's incorporating the additional claims in question. (23)

It is clear that a nonmentalistic interpretation of Chomsky's wh-explanation can be taken to be a set of claims $A$, a mentalistic interpretation to be a set of claims $B$, and mentalistic claims such as ( 8$)(a)$ and (b) to be the additional claims incorporated in $B$. Moreover, for Chomsky's mentalistic claims ( 8$)(a)$ and (b) to have real empirical content, there must in principle be evidence which would bear on them but which would simply be irrelevant to the validation of the claims incorporated in a nonmentalistic interpretation of the wh-explanation. In the absence of such evidence, these mentalistic claims would be evidentially indeterminate and the difference between Chomsky's mentalistic interpretation of the wh-explanation and a nonmentalistic interpretation of it, would be either terminological or metaphysical. The question, then, is whether there is or is not evidence which, in this sense, shows that the mentalistic claims in question are evidentially determinate and, thus, empirical. 
Intuitive evidence --- or as Chomsky (1976:12) calls it "evidence derived from informant judgment" -_ clearly does not show mentalistic claims to be empirical. For, as is made clear by Katz (1977:565), this kind of evidence is just as relevant to the validation of nonmentalistic hypotheses as it is to the validation of mentalistic hypotheses. And this takes us to the heart of the matter: Chomsky's Nonnecessity Thesis allows the mentalist in principle to derive all the evidence he needs for the validation of mentalistic claims from informant judgments alone. This thesis, thus, effectively destroys the basis of an argument to the effect that there is an empirical difference between Chomskyan mentalism and (a form of) nonmentalism. For, if mentalistic claims have to be empirical claims, they must be responsive to a kind of evidence which is irrelevant in principle to the validation of nonmentalistic linguistic hypotheses. The fundamental problem with The Nonnecessity Thesis then is that, in an unqualified form, it reduces mentalistic claims such as (8)(a) and (b) either to mere verbalisms or to metaphysical speculations. The same point may be put differently: for ontological claims such as $(8)(a)$ and (b) to have empirical content, Chomsky's Nonnecessity Thesis must be replaced by a Necessity Thesis. The latter thesis would state that mentalistic claims could not be validated without recourse to one or another kind of nonintuitive evidence which was in principle irrelevant to the validation of nonmentalistic hypotheses.

Notice that the adoption of a Necessity Thesis aggravates the problem which was denoted above by means of the expression "The Mentalist-Rationalist Paradox". In terms of the idealizations considered above, on the one hand, evicence not derived from genuine intuitions is disallowed in principle from being used to validate mentalistic claims. In terms of a Necessity Thesis, on the other hand, mentalistic claims must be validated with reference to such nonintuitive evidence in order to be empirical.

\section{The nonprivileged status of external evidence}

Chomsky's third fundamental evidential thesis may be reconstructed as follows: 
(9) The Nonprivileged Status Thesis: External evidence derived from such performance phenomena as production, recognition, recall, and language use in general may bear on mentalistic hypotheses, but (when it does) has no privileged status in relation to intuitive evidence.

This thesis represents the gist of the following remarks by Chomsky (1976: 11-12): "Suppose now that someone were to devise an experiment to test for the presence of a wh-clause in underlying representations --- let us say, a recognition or recall experiment. Or let us really let down the bars of imagination and suppose that someone were to discover a certain pattern of electrical activity in the brain that correlated in clear cases with the presence of wh-clauses: relative clauses (finite and infinitival) and wh-questions (direct and indirect). Suppose that this pattern of electrical activity is observed when a person speaks or understands (1) $[=\operatorname{our}(1)$ above -- R.P.B. $]$. Would we now have evidence for the psychological reality of the postulated mental representations?

"We would now have a new kind of evidence, but I see no merit to the contention that this new evidence bears on psychological reality whereas the old evidence only related to hypothetical constructions. The new evidence might or might not be more persuasive than the old; that depends on its character and reliability, the degree to which the principles dealing with this evidence are tenable, intelligible, compeliing, and so on. In the real world of actual research on language, it would be fair to say, I think, that principles based on evidence derived from informant judgment have proven to be deeper and more revealing than those based on evidence derived from experiments on processing and the like, but the future may be different in this regard. If we accept --- as I do --- Lenneberg's contention that the rules of grammar enter into the processing mechanisms, then evidence concerning production, recognition, recall, and language use in general can be expected (in principle) to have bearing on the investigation of rules of grammar, on what is sometimes called 'linguistic competence' or 'knowledge of language'. But such evidence, where it is forthcoming, has no privileged character and does not bear on psychological reality in some unique way. Evidence is not subdivided into two categories: evidence that bears on reality and evidence that just confirms or 
refutes theories (about mental computation and mental representations, in this case). Some evidence may bear on process models that incorporate a characterization of linguistic competence, while other evidence seems to bear on competence more directly, in abstraction from conditions of language use. And, of course, one can try to use data in other ways. But just as a body of data does not come bearing its explanation on its sleeve, so it does not come marked 'for confirming theories' or 'for establishing reality'."

Many of the quoted remarks by Chomsky appear to be quite sound. For example, it cannot be disputed that the weight of external evidence depends on its relevance, reliability, and the theoretical principles involved in its interpretation. Moreover, such evidence cannot demonstrate the truth of the mentalistic claims on which it positively bears; a point to which we return in $\$ 9$ below. Chomsky's Nonprivileged Status Thesis nevertheless has various questionable aspects, of which we shall consider the two most important ones.

\subsection{Ontological indeterminacy}

The first questionable aspect of The Nonprivileged Status Thesis relates to the ontological indeterminacy of Chomskyan mentalistic claims. A theoretical claim is ontologically determinate if the entity postulated by it or the state of affairs described by it is uniquely identifiable in the real world on which the claim is intended to bear. To require that an entity or state of affairs have to be uniquely identifiable is to require that the general nature and specific properties of this entity or state of affairs must be so clear that the entity or state of affairs can be unambiguously recognized as such in terms of what is known about its nature and properties. This requirement does not entail that the entity or state of affairs has to be directly observable. The requirement must be so construed that it allows for the possibility that an entity or state of affairs which is not directly observable can be uniquely identified via its causal effects.

These points may be illustrated with reference to the following two physical claims from Bahcall and Davis's (1976:264) discussion of problems in 
the theory of how the sun and other stars produce their radiant energy:

(10) (a) The sun's heat is produced by thermonuclear reactions that fuse light elements into heavier one, thus converting mass into energy.

(b) The basic solar process is the fusion of four protons to form an alpha particle, two positrons $\left(\underline{e}^{+}\right)$, and two neutrinos ( $\underline{v})$; that is, $4 \underline{p} \rightarrow \underline{\alpha}+2 \underline{e}^{+}+2 \underline{v}$.

As ontological claims, these physical claims are ontologically highly determinate. They describe a physical state of affairs which is uniquely identifiable. That is, the properties of theoretically postulated entities such as "sun", "solar process", "light element", "heavier element", "proton", "alpha particle", "positron", and "neutrino" are so clear to (astro-)physicists that they are able to recognize each of these entities as such on the basis of their knowledge of its properties. That is, the theoretical physical concepts in question have uniquely identifiable referents in a real physical world. As pointed out by Bahcall and Davis (1976:264), the process described by the physical claims (10)(a) and (b) can even be reproduced experimentally in terrestrial fusion reactors. Moreover, the description given by these two claims can be made highly precise: the reactions produced by the basic solar process can be quantified, as in fact they are by Bahcall and Davis (1976:265).

Consider now once again Chomsky's two mentalistic claims (8)(a) and (b). As ontological claims, these claims are quite indeterminate compared to the physical claims $(10)(a)$ and (b). What uniquely identifiable referents do linguistic concepts such as "PRO", " $t$ ", "wh", "(wh-)movement", "(wh-)island", "(wh-island) constraint", etc. have in a real mental world? It is not even clear what the general make-up of such a mental world would be. Thus, Chomsky fails to specify what entities or states of affairs would correspond in such a world to "a computation" or "a representation". As Chomsky uses the expressions "computation" and "representation" within this context, they are at best metaphors, at worst completely contentless. (25) It is therefore not strange that it would smack of science fiction to talk of experimentally reproducing the mental state of affairs described by the mentalistic claims ( 8$)(a)$ and $(b)$. And, not unexpectedly, 
Chomsky gives no indication of what it would entail to quantify these mentalistic claims. In sum: compared to physical claims such as (10) (a) and (b), Chomskyan mentalistic claims are ontologically highly indeterminate.

Now, theoretical claims which are assigned the status of ontological claims but which are ontologically indeterminate fail to be empirical in the conventional sense of 'not refutable/falsifiable in principle'. For an ontological claim to be refutable in principle it must, first of all, be possible to identify in the real world an entity or state of affairs which is clearly the intended referent of this claim. Moreover, it must be possible to ascertain whether this entity or state of affairs does or does not have the property or properties the claim attributes to it. In the case of ontologically indeterminate claims, it is just not clear what entities or states of affairs in a real world would, if they existed at all, have properties that were not the ones these claims attributed to them. And this appears to be true of Chomskyan mentalistic claims such as ( 8 )(a) and (b). Notice that it is, by implication, not claimed that a nonmentalistic interpretation of the wh-explanation is nonempirical as well. The general point is that, for it to be empirical, a mentalistic interpretation of this explanation must meet more stringent conditions than a nonmentalistic interpretation has to meet.

What now can be done in order to reduce the ontological indeterminacy of the mentalistic claims under consideration? A first necessary step is to strip Chomsky's expressions"to impute existence to theoretical constructs" and "to attribute psychological reality to theoretical constructs" of their obscurity. A natural way of doing this entails developing one or more ontological conditions for (the entities postulated by) theoretical constructs involved in the mentalistic claims. Such conditions would specify the circumstances under which it would be proper to claim that an arbitrary theoretically postulated mental entity did or did not exist. Let us consider two examples of putative ontological conditions of this kind.

A first ontological condition for theoretical linguistic constructs may 
be derived from a particular view which Chomsky has repeatedly put forward. This is the view that linguistic competence, as described by a generative grammar, is in fact used in one way or another in linguistic performance. In the article of his under consideration here, Chomsky (1976:12) presents this view as follows: "If we accept --- as I do --Lenneberg's contention that the rules of grammar enter into the processing mechanisms ...". And in their recent joint paper, Chomsky and Lasnik (1977:427), having related the linguist's grammar to the child's grammar, state that "The grammar $G$ is embedded in a performance system that enables knowledge of language (competence) to be put to use in speech and understanding". (26)

From this view of Chomsky's it is possible to derive in a natural manner the following ontological condition.

(11) The Performance Condition: A theoretically postulated mental entity cannot be granted existence or psychological reality unless it "is put to use in speech and understanding" or unless it "enters into the processing mechanisms".

This ontological condition has to be clarified in various respects. For example, the content of the expressions "is put to use" and "enters into" must be explicated in clear, nonambiguous terms.

As formulated above, The Performance Condition is nevertheless sufficientIy precise to illustrate the way in which the content of Chomsky's expressions "impute existence to" or "attribute psychological reality to" may be clarified with a resulting increase in the determinacy of Chomskyan mentalistic claims. In terms of The Performance Condition the expressions "to impute existence to $X^{\prime \prime}$ and "to attribute psychological reality to $X^{\prime \prime}$ have the meaning "to claim that $X$ is used in actual speech and understanding" or "to claim that $\mathrm{X}$ enters into the processing mechanisms".

The adoption of The Performance Condition has rather clear implications for the evidence needed for validating mentalistic claims. In terms of this condition, evidence about the use or non-use in performance of a particular theoretically postulated mental entity becomes crucial to the validation of the mentalistic claim which postulates the existence of this 
entity. To put it differently, The Performance Condition assigns a privileged status to (certain kinds of) performance evidence in the validation of mentalistic claims about an idealized competence.

It appears to me that many scholars have been under the impression that Chomsky in fact accepts The Performance Condition. It is these scholars whose views Chomsky (1976:6) characterizes as follows: "The literature takes a rather different view. Certain types of evidence are held to relate to psychological reality, specifically, evidence deriving from studies of reaction time, recognition, recall, etc. Other kinds of evidence are held to be of an entirely different nature, specifically, evidence deriving from informant judgments as to what sentences mean, whether they are well formed, and so on. Theoretical explanations advanced to explain evidence of the latter sort, it is commonly argued, have no claim to psychological reality, no matter how far-reaching, extensive, or persuasive the explanations may be, and no matter how firmly founded the observations offered as evidence. To merit the attribution of 'psychological reality', the entities, rules, processes, components, etc. postulated in these explanatory theories must be confronted with evidence of the former category".

Chomsky (1976:6, 12) goes on, however, to reject the view which assigns a privileged status to evidence from performance. Thus he (1976:12) states that "... evidence concerning production, recognition, recall, and language use in general can be expected (in principle) to have bearing on the investigation of rules of grammar, on what sometimes is called 'Iinguistic competence' or 'knowledge of language'. But such evidence, where it is forthcoming, has no privileged character and does not bear on psychological reality in some unique way".

These remarks by Chomsky give rise to a serious problem. In order to deny evidence from performance a privileged status, Chomsky has to reject The Performance Condition. For, it is by virtue of this condition that performance evidence has a privileged status. By rejecting The Performance Condition, however, Chomsky would make a mystery of his view that linguistic competence "enters into processing mechanisms" or is "put to use in speech and understanding". For, The Performance Condition follows in a natural manner from Chomsky's views on the use of competence in actual 
performance. And, even more important, the rejection of The Performance Condition exposes Chomskyan mentalism anew to the challenge that it is nothing more than a terminological game or a metaphysical system. Recall that the function of The Performance Condition, as an ontological condition, is to enhance the ontological determinacy of mentalistic claims sufficiently to render these claims refutable.

The Performance Condition, of course, is not the only ontological condition that can fulfil this function. It may be argued that this condition should be replaced, or accompanied, by the following one:

$$
\begin{aligned}
& \text { The Physical Basis Condition: A theoretically postulated } \\
& \text { mental entity cannot be granted existence or psychological } \\
& \text { reality, unless it is somehow realized in the (physical) } \\
& \text { mechanisms of the brain. }
\end{aligned}
$$

This further ontological condition is intimated in such statements by Chomsky (1976:3) as the following: "Ultimately we hope to find evidence concerning the physical mechanisms that realize the program, and it is reasonable to expect that results obtained in the abstract study of the system and its operation should contribute significantly to this end (and in principle, conversely)". The core of this view of Chomsky's is also to be found in an earlier paper by himself and Katz (1974:364): "... the grammar in the form it would take in models of speech production and perception must structurally correspond to some features of brain mechanism". From the latter quote it is clear that the physical mechanisms provided for in The Physical Basis Condition take on the form of neurophysiological entities and processes. (27)

Notice that if The Physical Basis Condition were to be adopted, then (also) neurophysiological evidence would have a privileged status in the validation of mentalistic claims. This condition contributes to the ontological determinacy of mentalistic claims -- and, by implication, reduces the verbalistic or metaphysical nature of such claims --- by assigning the expression "to impute existence to $X$ " the content "to claim that $X$ is realized neurophysiologically".

Chomsky may reject The Physical Basis Condition as well, thereby denying 
neurophysiological evidence a privileged status. Such a rejection, however, would leave him with problems analogous to those created by the rejection of The Performance Condition. First, claims such as the ones quoted above on the physical basis of grammars would become obscure. Second, mentalistic claims would remain ontologically indeterminate and, consequently, nonempirical.

This brings us to the crux of the matter: in order to make his mentalistic claims ontologically determinate enough that they will be empirical, Chomsky cannot do without ontological conditions such as The Performance Condition or The Physical Basis Condition. Whether or not the appropriate ontological conditions is/are one of these two conditions and/or one or more other conditions is immaterial to the argument. What is crucial is the fact that there is a need for conditions of this sort. But adopting one or more of these ontological conditions implies assigning a privileged status to one or more kinds of external evidence. Chomsky's Nonprivileged Status Thesis, however, by implication makes it impossible in principle to adopt any of these ontological conditions. Consequently, this thesis blocks the way to making Chomskyan mentalistic claims ontologically more determinate and, thus, empirical. This, then, is one of the two principal ways in which The Nonprivileged Status Thesis is questionable.

The conclusion that Chomskyan mentalism has to adopt one or more ontological conditions bears directly on The Mentalist-Rationalist Paradox. In terms of such conditions, the Chomskyan mentalist would have to use external evidence for validating mentalistic claims. This mentalist, moreover, would have to assign a privileged status to certain kinds of this evidence. As a result, the conflict between his mentalistic ontology and his rationalistic : phenomenology would become even more significant.

\section{2 "Reality" vs. "mental computation and mental representations"}

The other questionable aspect of The Nonprivileged Status Thesis concerns its relation to a particular distinction drawn by Chomsky. Recall that, in his defence of The Nonprivileged Status Thesis, Chomsky (1976:12) makes the following statements: "But such evidence [i.e. performance evidence -.. R.P.B.], where it is forthcoming, has no privileged character and 
does not bear on psychological reality in some unique way. Evidence is not subdivided into two categories: evidence that bears on reality and evidence that just confirms or refutes theories (about mental computation and mental representations, in this case)". Appropriately interpreted, Chomsky's statement that evidence is not subdivided into two categories is correct. The appropriate interpretation is not, however, the one he seems to envisage. For, the two categories of evidence he alludes to are based on an untenable distinction: "reality vs. mental computation and mental representations". Within the framework of Chomsky's mentalism, it is far from being clear in what nonbizarre sense "reality" is distinct from "mental computation and mental representations". That is, it is not clear in what sense a "mental computation" or "mental representation" can be anything other than one of the things of which this "reality" is made up. So there cannot be two kinds of evidence: "evidence that bears on reality" as opposed to "evidence that just confirms or refutes theories (about mental computation and mental representations)".

The distinction involved in The Nonprivileged Status Thesis is, however, a different one. This is the distinction between, on the one hand, evidence bearing on a reality which includes "mental computation and mental representations" and, on the other hand, evidence that does not bear on this reality at all. The discussion above has made it clear that evidence of the former kind, viz. external evidence, does have a privileged status in regard to evidence of the latter kind, viz. intuitive evidence. It has been shown that intuitive evidence cannot serve as a basis on which to distinguish between Chomskyan mentalism and a nonmentalistic view of linguistic theories. Thus the Nonprivileged Status Thesis cannot derive any justification from the distinction drawn in the quoted remarks by Chomsky. This is not the distinction pertinent to the adoption of a Privileged Status Thesis. (28)

\section{The Nonconclusiveness of external evidence}

Chomsky's fourth evidential thesis does not bear directly on The Mentalist -Rationalist Paradox. However, to gain a fuller understanding of his position on the role of external evidence in the validation of mentalistic claims, it is necessary to consider this thesis briefly: 
The Nonconclusiveness Thesis: The external evidence which derived from performance data, for example --- can be furnished in support of a mentalistic claim cannot conclusively show this claim to be true.

This thesis reconstructs the essence of remarks such as the following by Chomsky (1976:5-6): "Or, like the astronomer dissatisfied with the study of light emissions from the periphery of the sun, we can search for more conclusive evidence, always aware that in empirical inquiry we can at best support a theory against substantive alternatives and empirical challenge, not prove it to be true".

The Nonconclusiveness Thesis and the quoted remarks on which it is based appear, for the most part, to be beyond serious dispute. This thesis places severe limitations on the weight and potential usefulness of external evidence in the validation of mentalistic claims. For, what point would there be to appealing to such evidence if this evidence could not in principle contribute significantly to the support for mentalistic claims. There are, however, two respects in which Chomsky's discussion of the nonconclusive nature of external evidence is less than adequate. And these inadequacies reflect negatively on The Nonconclusiveness Thesis.

First, Chomsky fails to make clear that the nonconclusiveness of external evidence does not spring primarily from the external nature of such evidence. The nonconclusiveness of this kind of evidence springs from the fact that scientists in general do not have at their disposal any form of argument for demonstrating the truth of empirical hypotheses. (29) The forms of argument which can be used to justify empirical hypotheses are nondemonstrative by their very nature. And these forms of argument have the property of nondemonstrativeness regardless of the kind of evidence furnished, within the framework of them, in support of empirical hypotheses. In fact, therefore, The Nonconclusiveness Thesis conveys no information at all about external evidence as a distinct kind of evidence.

Second, Chomsky fails to make clear that external evidence will have greater weight when used as negative evidence for refuting mentalistic claims, than when used as positive evidence for confirming such claims. Although it is impossible to refute any theory of some complexity conclu- 
sively, (30) the form of argument pertinent to such refutation is demonstrative. (31) It is the demonstrative nature of this form of argument which will add to the weight of the external evidence --- or, for that matter, any kind of evidence --- used within the framework of it for refuting mentalistic claims.

In the article under consideration, Chomsky presents a quite unbalanced view of the validation of empirical claims. He depicts this validation as if it consisted almost entirely in providing support for such claims, furnishing justification for them, or demonstrating their truth. For all practical purposes the component of refutation, which is essential to such validation, is ignored by Chomsky. (32) This, in sum, is the reason why The Nonconclusiveness Thesis is inaccurate as an assessment of the potential weight of external evidence in the validation of mentalistic claims.

\section{Summary and conclusion}

Reconstructed in the form of explicit evidential theses, Chomsky's position on the nature of the evidence pertinent to the validation of mentalistic linguistic claims turns out to be untenable. The first evidential thesis, The Varied Sources Thesis, allows for many and varied sources of evidence for validating such claims. However, the rationalistic idealizations employed by Chomsky for the purpose of limiting the domain of linguistic theories restrict these sources of evidence to one only, viz. native speaker's linguistic intuitions. The conflict between the former thesis and the consequences of the latter idealizations clearly represents a clash between a mentalistic and a rationalistic principle within the overall Chomskyan approach.

The paradoxical nature of this conflict is highlighted by a basic defect in Chomsky's second evidential thesis, The Nonnecessity Thesis. The adoption of this thesis --- which states that it is not necessary to use, in addition to intuitive (= internal) evidence, other, i.e. nonintuitive (= external), evidence for the validation of mentalistic claims -.. renders these claims evidentially indeterminate. Consequently, as ontological claims, they are nonempirical. For (sets of) mentalistic claims to be empirically distinct from related (sets of) nonmentalistic claims, 
external evidence must be brought to bear on the former claims. This entails that The Nonnecessity Thesis should be replaced by a Necessity Thesis. As a result, the above-mentioned Mentalist-Rationalist Paradox takes on even greater significance: whereas the rationalistic idealizations employed by Chomsky disallow external evidence in the validation of mentalistic claims, a Necessity Thesis would specify that these claims would be nonempirical unless they were made responsive to external evidence.

The conflict which gives rise to the Mentalist-Rationalist Paradox is aggravated by a flaw in Chomsky's third evidential thesis. This thesis, The Nonprivileged Status Thesis, specifies that the external evidence that bears on mentalistic claims does not have a privileged status relative to intuitive evidence. To enhance the ontological determinacy of Chomskyan mentalistic claims to such an extent that they become refutable in principle, one or more ontological conditions have to be adopted. The function of these conditions is to strip Chomsky's expressions "to impute existence to" and "to attribute psychological reality to" of their obscurity. The Nonprivileged Status Thesis, however, disallows the adoption of such conditions because they would automatically assign a privileged status to one or more kinds of external evidence. Consequently, the Nonprivileged Status Thesis has to be replaced by a Privileged Status Thesis. The basic claim of such a thesis, however, is in direct conflict with the abovementioned rationalistic idealizations.

Chomsky's fourth evidential thesis, The Nonconclusiveness Thesis, asserts that external evidence cannot show the mentalistic claims on which it bears to be true. By implication, the role of such evidence in the validation of mentalistic claims is played down. Though this thesis appears at first glance to express an undisputable truth, it is nevertheless misleading. It depicts a defining property of confirmation, viz. the property of nondemonstrativeness, as if it were a property peculiar to external evidence. Moreover, this thesis is based on a view of the logic of validation which fails to explicitly take into account that this logic has, in addition to confirmation, a second component, viz. refutation. Consequently, The Nonconclusiveness Thesis diverts the attention from the important role which external evidence may play in the refutation of mentalistic hypotheses. Chomsky's Nonconclusiveness Thesis does not bear directly on 
The Mentalist-Rationalist Paradox. The fact that this thesis is unacceptable, however, further undermines Chomsky's general position on the nature of the evidence pertinent to the validation of mentalistic claims.

Against this background, we can now reconsider Chomsky's question of whether it is or is not "legitimate" and "proper". to "impute existence to" or "attribute psychological reality to" the theoretical constructs of linguistic theories. We have seen that this imputing of existence to theoretical linguistic constructs would be "illegitimate" and "improper" if it would lead to the generation of nonempirical mentalistic claims. Moreover, it has become clear that Chomsky's Nonnecessity Thesis and Nonprivileged Status Thesis render his mentalistic claims, as ontologic claims, nonempirical. Consequently, these theses have to be replaced by a Necessity Thesis and a Privileged Status Thesis, respectively. The adoption of the latter two theses, however, would contribute significantly to The Mentalist-Rationalist Paradox. Thus, to legitimate the imputing of existence to theoretical linguistic constructs this paradox would have to be resolved. (34) 
FOOTNOTES

This paper deals with one aspect of a cluster of problems which I discuss in more detail elsewhere -- cf. Botha to appear a, b, cl. David Lightfoot and Walter Winckler have subjected these papers to penetrating criticism. They are, of course, not to be held responsible for the limitations of the present paper. Walter Winckler, in addition, has suggested many useful improvements to the formulation of the former papers.

1. Cf., e.g., Botha 1971:ch.4; 1973:ch.4; Cooper 1975; Derwing 1973: ch.8; Derwing and Harris 1975; Schwartz 1969; Steinberg 1975; Stich 1975.

2. Thus consider the following remarks by Katz (1977:564): "The competencist might thus introduce principle $R$ as the counterpart to $A$.

(R) A grammar of a language must be psychologically real in the sense that it represents an idealization of the knowledge that speakers of a language have about its grammaticall structure, that is, it represents an ideal of their knowledge in the sense of $M$.

This, however, is not the only possibility. The competencist might not wish to impose such a constraint, but only to claim that an optimal grammar in the above sense is (as a matter of fact) psychologically real in the sense of being an idealization of actual speaker-hearer's grammatical knowledge. It is, I think, not clear which of these possibilities Chomsky takes ...." In this quote $A$ denotes the following condition: "Semantic representations are psychologically real in the sense that, given appropriate idealizations, understanding a sentence requires the recovery of its semantic representation" (Katz 1976:560). M, by contrast, refers to the following methodological principle: "As real conditions more closely approximate to ideal ones, the predictions of the laws formulated over ideal objects must approximate to actual observations more and more closely" (Katz 1976:563). 
3. This is their 1974 paper "What the linguist is talking about".

4. Chomsky (1976:8) clarifies certain aspects of this representation in the following note: "Take $t$ to be the 'trace' left by movement of which from the position where $t$ appears in (12) $[=$ our (4) -R.P.B.] , in accordance with the trace theory of movement rules ... Take PRO to be an abstract "pronominal" form, which can be regarded as an uncontrolled trace ...."

5. In a later paper, Chomsky ( $1978 \mathrm{~b}: 16 \mathrm{ff}$. ) attempts to reduce the whisland constraint to a more general principle, the principle of subjacency. This, however, is irrelevant to the present discussion.

6. The distinction between a (fragment of) nonmentalistic grammar and a corresponding (fragment of) mentalistic grammar is considered in more detail in $\$ 4$ below.

7. Within a more comprehensive study of mentalistic linguistics, Chomskyan mentalism will have to be distinguished from other forms of mentalism, for example the mentalism of Katz (1977), the mentalism of Bresnan (1976), etc.

8. It will become clear in $\hat{S}_{5}$ below that the idealizations in question are in fact more powerful: they abstract away from the effects of noncognitive systems as well.

9. In later papers Chomsky (1978a; 1978b) repeats the essentials of the account given in this paragraph of the objects, aims and idealizations of mentalistic linguistic theories.

10. For a discussion of some of the problems involved in choosing such conditions in a nonarbitrary manner cf. Bartley 1968.

11. In a later paper, Chomsky (1978b) equates "empirical" with "falsifiable in principle". In $\mathbf{S}_{5.1}$ of Botha to appear c, however, it is shown that Chomsky fails to explicate the latter notion. 
12. The thermonuclear analogy is dealt with in Botha to appear a, the neurophysiological analogy in Botha to appear $c$.

13. Cf. e.g. Chomsky 1965:25ff. for the notion "primary linguistic data".

14. Some of these abstractions and idealizations have been considered in $\S_{3}$ above. We return to them in $\$ 6$ below.

15. Cf. Chomsky and Katz 1974:359-360.

16. Cf., e.g., Bever 1974, Katz and Bever 1977, Bever, Katz and Langendoen 1977.

17. Katz (1977:561) presents "competencism" as "... the classical Chomskyan position: it makes a strict competence/performance distinction, separating 'the speaker-hearer's knowledge of the language' from 'the actual use of language in concrete situations', and it takes a grammar 'to be the description of the ideal speaker-hearer's competence, not of the use of language' (Chomsky (1965:4))."

18. At this juncture it is sufficient to represent Bever's (1974:I78) quite general characterization of the methodological assumptions central to this philosophy of science:

"a) Specific factual phenomena are often the result of interactions among different (physical, psychological, biological) systems.

b) The formal theory in each system should be as limited as possible to be as testable as possible.

c) When a new fact can be described by two existing systems, but would require elaboration of one of them and not the other, the fact is interpreted as due to the system not requiring elaboration for its description."

19. This interpretation is borne out also by the following remarks of Chomsky's (1976:12): "... evidence concerning production, recognition, recall, and language use in general can be expected (in prin- 
ciple) to have a bearing on the investigation of rules of grammar, on what is sometimes called 'Iinguistic competence' or 'knowledge of language'. But such evidence, where it is forthcoming, has no privileged character and does not bear on psychological reality in some unique way". The content of this quote is analyzed more closely in S8 below.

20. Notice, incidentally, in regard to the quote given above that it is difficult to reconcile Chomsky's "can do no more" formulation in the first statement with the "Or" formulation of the final statement. How can a linguist have the option of doing B (the "Or" formulation) if he "can do no more" than A?

21. A further problem with this thesis concerns the manner in which it is related to Chomsky's thermonuclear analogy mentioned in 84 above. This problem is dealt with in Botha to appear b:\$4.1.

22. In the "conventional" sense a claim is empirical if (a) its content is so clear that it has precise test implications; (b) there is, in principle, available "empirical" data with which these test implications may be confronted; (c) it is not protected by means of ad hoc devices from refutation. A claim that meets these conditions is said to be refutable or falsifiable in principle. Chomsky (1978b:9) also equates "empirical" to "falsifiable in principle".

23. Note that evidential indeterminacy should be sharply distinguished from underdeterminedness by evidence. All empirical claims are underdetermined by the evidence that bears positively on them. This is so because positive evidence does not demonstrate or prove the claim(s) on which it bears to be true. Whereas an empirical claim is in principle underdetermined by the evidence bearing positively on it, a claim that is evidentially indeterminate is in principle a nonempirical claim.

24. It is on this discussion by Bahcall and Davis (1976) that Chomsky bases the thermonuclear analogy mentioned above. 
25. Also in his paper "A theory of core gramar" (1978b:13, 16, 17, 22) Chomsky uses the expressions "mental computation(s)" and "mental representations" without attempting to specify what nonfigurative meaning "computation" and "representation" may have in this context. The (illusory) nature of Chomsky's mental entities is analyzed in greater depth in $\$ \S_{2}$ and 4 of Botha to appear $c$.

26. For a repetition of the essence of this view cf. also Chomsky and Lasnik $1977: 434$.

27. In $\$ 4.2$ of Botha to appear c. I formulate two analogues of The Physical Basis Condition.which apply to the mentalistic claims expressed in the general linguistic theory.

28. Such a condition is proposed in Botha to appear c:\$5.4.

29. Cf. Botha 1973: \$ీ\$ీ2.3-2.4 for a discussion of this point.

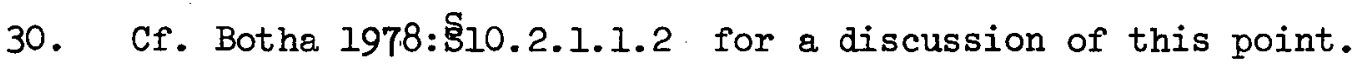

31. This point is explicated in Botha 1978:8110.2.1.2.1.

32. In Botha to appear a: $\$ \S 6 \cdot 2 .-6.4$ it is shown that Chomsky even goes so far as to project his justification-oriented. conception of the validation of empirical claims on to the falsification-oriented methodology of the form of physical inquiry practised by Bahcall and Davis.

33. In Botha to appear b:\$6.2 it is shown that even when Chomsky practices linguistic analysis he fails to take into account the role which external evidence may play in the refutation of linguistic hypotheses.

34. For one approach to resolving this paradox cf. Botha to appear c . 
REFERENCES

Bahcall, John N, and Raymond Davis, Jr. 1976

"Solar neutrinos: a scientific puzzle", Science, Vol. 191:264-267.

Bartley, W.

1968

\footnotetext{
"Theories of demarcation between science and metaphysics", in Lakatos and Musgrave (eds.) 1968:46-64.
}

Bever, Thomas G.

1974 "The ascent of the specious or there's a lot we don't know about mirrors", in Cohen (ed.) 1974:173-200.

Bever, Thomas G., Jerrold J. Katz, and D. Terence Langendoen

1977 Introduction to Bever, Katz and Langendoen (eds.) 1977: 1-9.

Bever, Thomas G., Jerrold J. Katz, and D. Terence Langendoen (eds.) 1977 An integrated theory of linguistic ability. Hassocks, Sussex: The Harvester Fress.

Botha; Rudolf P. 1971 Methodological aspects of transformational generative phonology (= Janua Linguarum, Series Minor, No.112) The Hague and Paris: Mouton Publishing Company.

Botha, Rudolf P. 1973

The justification of linguistic hypotheses. A study of nondemonstrative inference in transformational grammar (= Janua Linguarum, Series Maior, No.84). The Hague and Paris: Mouton Publishing Company.

Botha, Rudolf $P$. 1978

Generatiewe taalondersoek. 'n Sistematiese inleiding. Kaapstad/Pretoria: Hollandsch Afrikaansche Uitgevers Maatschappij (To be published in English under the title 
The conduct of linguistic inquiry: A systematic introduction to the methodology of generative grammar.)

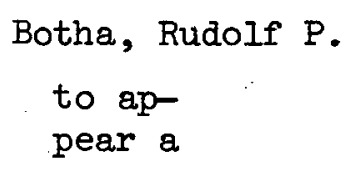

Bresnan, Joan

1976

Chomsky, Noam

1965

Aspects of the theory of syntax. Cambridge, Mass.: MIT Press.

Chomsky, Noam 1976

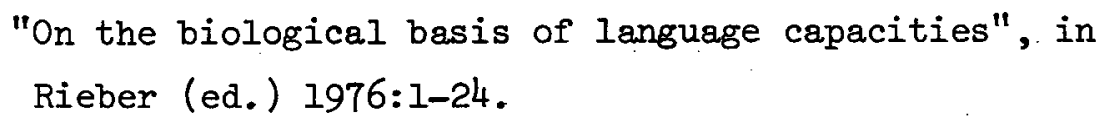

Chomsky, Noam

$1978 \mathrm{a}$

On binding. Mimeographed.

Chomsky, Noam

$1978 \mathrm{~b}$

"A theory of core grammar", Glot, Vol. 1:7-26.

Chomsky, Noam and Jerrold J. Katz 1974

"What the linguist is talking about", The Journal of Philosophy, Vol. LXXI:347-367.

Chomsky, Noam, and Howard Lasnik

1977

"Filters and control", Linguistic Inquiry, Vol. 8:425-504. 
Cohen, Darid (ed.)

1974

Explaining linguistic phenomena. Washington, D.C.: Hemisphere Publishing Corporation.

Cohen, David, and Jessica R. Wirth (eds.)

1975 Testing linguistic hypotheses. Washington, D.C.:

Hemisphere Publishing Corporation.

Cooper, David E.

1975

Knowledge of language. London: Prism Press.

Derwing, Bruce L.

1973

Transformational grammar as a theory of language acqui-

sition. A study in the empirical, conceptual, and

methodological foundations of contemporary linguistics.

Cambridge: At the University Press.

Derwing, Bruce L., and Peter R. Harris

1975 "What is a generative grammar?", in Koerner (ed.) 1975:

$297-314$.

Fodor, J.I., J.A. Fodor, and M.F. Garrett

1975

"The psychological unreality of semantic representations", Iinguistic Inquiry, Vol. 6:515-532.

Hook, Sidney (ed.)

1969 Language and philosophy. New York: New York University Press.

Katz, Jerrold J., and Thomas G. Bever

1977

"The fall and rise of empiricism", in Bever, Katz, and

Langendoen (eds.) 1977:11-64.

Katz, Jerrold J.

1977

"The real status of semantic representations", Linguistic Inquiry, Vol. 8:559-584.

Koerner, I.F.K. (ed.)

The transformational-generative paradigm in modern linguistic theory. Amsterdam: John Benjamins B.V. 
Lakatos, Imre, and Alan Musgrave (eds.)

1968 Problems in the philosophy of science. Amsterdam:

North-Holland

Rieber, R.W. (ed.)

1976 The neuropsychology of language. Essays in honor of

Eric Lenneberg. New York and London: Plenum Press.

Schwartz, Robert

1969 "On knowing a grammar", in Hook (ed.) 1969:183-190.

Steinberg, Danny D.

1975. "Chomsky: from formalism to mentalism to psychological invalidity", Glossa, Vol. 9:218-252.

Stich, Stephen P.

1975 "Competence and indeterminacy", in Cohen and Wirth (eds.) 1975: 93-109. 